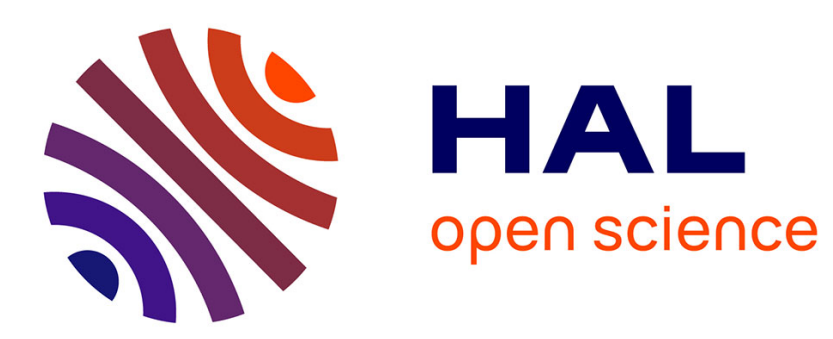

\title{
Approximating and Simulating Multivalued Stochastic Differential Equations
}

Dominique Lepingle, Thi Thao Nguyen

\section{To cite this version:}

Dominique Lepingle, Thi Thao Nguyen. Approximating and Simulating Multivalued Stochastic Differential Equations. Monte Carlo Methods and Applications, 2004, 10, pp.129-152. hal-00003500

\section{HAL Id: hal-00003500 \\ https://hal.science/hal-00003500}

Submitted on 9 Dec 2004

HAL is a multi-disciplinary open access archive for the deposit and dissemination of scientific research documents, whether they are published or not. The documents may come from teaching and research institutions in France or abroad, or from public or private research centers.
L'archive ouverte pluridisciplinaire HAL, est destinée au dépôt et à la diffusion de documents scientifiques de niveau recherche, publiés ou non, émanant des établissements d'enseignement et de recherche français ou étrangers, des laboratoires publics ou privés. 


\title{
Approximating and Simulating Multivalued Stochastic Differential Equations
}

\author{
Dominiqu LEPINGLE Thi Thao NGUYEN \\ MAPMO, Université d'Orléans, F-45067 Orléans Cedex2 \\ email: lepingle@labomath.univ-orleans.fr \\ email: tnguyen@labomath.univ-orleans.fr
}

\begin{abstract}
Abstact
We propose a two-step simulation scheme for the solution of a singular stochastic differential equation with exploding drift. First we estimate the strong order of the Yosida approximation. Then we use a semi-implicit Euler scheme to discretize the approximate solution. Numerical experiments are displayed for the paths of Brownian particles with strong repulsive interaction. We also present two simple simulation schemes for Bessel processes with arbitrary dimension.
\end{abstract}

Keywords: Multivalued stochastic differential equations, Yosida approximation, interacting Brownian particles, semi-implicit scheme.

\section{Introduction}

Multivalued stochastic differential equations are used to model a large class of random evolutions such as stochastic differential equations with reflection on the boundary of a domain [22], diffusion equations with hysteresis [16], or systems of Brownian particles with repulsive interaction [10]. Existence and approximation of such equations, also called stochastic variational inequalities, have received much attention in the recent years. Existence has been proved in several papers ([3], [8], [9], [21]). Numerical approximation has been tackled in [1], [4] and [19].

The last two authors have proposed the same projection scheme which is natural and efficient in the case of pure reflection ([20], [18]). In [19], the convex function is assumed to be continuous on the closure of the domain and in [4] its gradient satisfies a polynomial growth condition. In both papers, the difficult case of exploding drift is avoided. In the work [1], these restrictions are released and the authors use a splitting up method. Their scheme is not easy to implement because at each step they have to solve a multivalued ordinary differential equation. Our aim is to set a discrete simulation scheme which also works for stiff equations. An example of such equations is given in dimension one by the Bessel processes. Let us mention the recent work [13] where the weak error of an approximation scheme for these processes is estimated.

The paper is organized as follows. In Section 2, we recall some material on multivalued maximal monotone operators associated with a proper lower semi-continuous convex function to introduce multivalued stochastic differential equations and their approximate equations. In Section 3, we estimate the strong order of approximation: note that in [21] the estimation 
order is $\varepsilon^{1 / 24}$ and in [1] it is $\varepsilon^{1 / 16}$. Improving the arguments in [21], we obtain $\varepsilon^{1 / 8}$. In Section 4, we cannot use an explicit Euler scheme to discretize the approximate solution because the drift term is Lipschitz continuous with constant $1 / \varepsilon$. So we resort to a more involved semiimplicit scheme which behaves much better, as was noted for stiff equations in [14]. Section 5 is devoted to the simulation of some systems of Brownian particles with repulsive interaction which are multidimensional extensions of Bessel processes.

\section{Preliminaries}

The purpose of this section is to recall some results on the subdifferentials of convex lower semi-continuous functions being maximal monotone operators which will be used all along this work. The relevant material on convex analysis and maximal monotone operators may be found in V. Barbu and Th. Precupanu [2] or in H.Brézis [6].

\subsection{Subdifferential of a convex function}

Let $\varphi$ be a convex function defined in $\mathbb{R}^{d}, d \in \mathbb{N}^{*}$. We denote by

$$
\operatorname{dom}(\varphi)=\left\{x \in \mathbb{R}^{d}: \varphi(x)<\infty\right\}
$$

the domain of $\varphi$. We say that $\varphi$ is proper if $\operatorname{Int}(\operatorname{dom}(\varphi)) \neq \emptyset$.

The subdifferential of $\varphi$, written as $\partial \varphi$, is an operator in $\mathbb{R}^{d}$ defined by its graph

$$
(x, y) \in \operatorname{Gr}(\partial \varphi) \Leftrightarrow \forall z \in \mathbb{R}^{d}, \varphi(x) \leq \varphi(z)+\langle y, x-z\rangle
$$

For any $x \in \mathbb{R}^{d}$, we note

$$
\partial \varphi(x)=\left\{y \in \mathbb{R}^{d}:(x, y) \in G r(\partial \varphi)\right\}
$$

The domain of $\partial \varphi$ is

$$
D(\partial \varphi)=\left\{x \in \mathbb{R}^{d}: \partial \varphi(x) \neq \emptyset\right\}
$$

The following proposition is stated without proof.

Proposition 2.1 The subdifferential $\partial \varphi$ is a multivalued maximal monotone operator in $\mathbb{R}^{d}$. Moreover, we have

$$
\operatorname{Int}(D(\partial \varphi))=\operatorname{Int}(\operatorname{dom}(\varphi)) \subset D(\partial \varphi) \subset \operatorname{dom}(\varphi) \subset \overline{\operatorname{dom}(\varphi)}=\overline{D(\partial \varphi)}
$$

where we recall that Int $(D)$ and $\bar{D}$ are respectively the interior and the closure (for the Euclidean topology) of $D$ in $\mathbb{R}^{d}$. 


\section{Particular case: Subdifferential of the indicator function.}

Let $D$ be a convex closed subset of $\mathbb{R}^{d}$ with nonempty interior. It follows that

$$
\mathbb{I}_{D}(x)= \begin{cases}0 & \text { if } \quad x \in D, \\ +\infty & \text { if } \quad x \notin D\end{cases}
$$

is convex, l.s.c. and proper with $\operatorname{dom}\left(\mathbb{I}_{D}\right)=D$. Its subdifferential is

$$
\partial \mathbb{I}_{D}(x)=\left\{y \in \mathbb{R}^{d}:\langle y, z-x\rangle \geq 0, \forall z \in D\right\},
$$

i.e.

$$
\partial \mathbb{I}_{D}(x)= \begin{cases}\emptyset & \text { if } \quad x \notin D, \\ \{0\} & \text { if } \quad x \in \operatorname{Int}(D), \\ \Pi_{x} & \text { if } \quad x \in \partial D,\end{cases}
$$

where $\Pi_{x}$ is the normal cone at $x$.

Note: In the one-dimensional case, every multivalued maximal monotone operator $A$ with $\operatorname{Int}(D(A)) \neq \emptyset$ is the subdifferential of a proper l.s.c. convex function. This property does not hold in the multidimensional case.

\subsection{Yosida approximation}

We will construct in this part a sequence of single valued approximations for the subdifferential of a proper 1.s.c. convex function. Let us recall that, for each $\varepsilon>0$ and $x \in \mathbb{R}^{d}$, the equation $x \in(I+\varepsilon A)(y)$ has one and only one solution $y$ in the domain of $A$ if $A$ is a maximal monotone operator of $\mathbb{R}^{d}$. The Yosida approximation of the subdifferential $\partial \varphi$ of a proper 1.s.c. convex function $\varphi$ is the application $\beta_{\varepsilon}$ defined by

$$
\beta_{\varepsilon}=\frac{1}{\varepsilon}\left(I-\pi_{\varepsilon}\right),
$$

where $\pi_{\varepsilon} x$ is the unique solution of the equation $y \in(I+\varepsilon \partial \varphi)^{-1}(x), x \in \mathbb{R}^{d}$.

Proposition 2.2 For each $\varepsilon>0$, we have:

i) $\pi_{\varepsilon}$ is a contraction from $\mathbb{R}^{d}$ to $D(\partial \varphi)$;

ii) $\beta_{\varepsilon}$ is a single valued maximal monotone operator defined on the whole $\mathbb{R}^{d}$, Lipschitz continuous with constant $\frac{1}{\varepsilon}$;

iii) for every $x \in \mathbb{R}^{d}, \beta_{\varepsilon}(x) \in \partial \varphi\left(\pi_{\varepsilon} x\right)$.

Proposition 2.3 For each $\varepsilon>0$, put

$$
\varphi_{\varepsilon}(x)=\min _{y \in \mathbb{R}^{d}}\left(\frac{1}{2 \varepsilon}|x-y|^{2}+\varphi(y)\right), x \in \mathbb{R}^{d} .
$$

Then $\varphi_{\varepsilon}$ is called the Yosida approximation of the function $\varphi$ and

i) $\varphi_{\varepsilon}: \mathbb{R}^{d} \rightarrow(-\infty,+\infty)$ is convex with domain $\operatorname{dom}\left(\varphi_{\varepsilon}\right)=\mathbb{R}^{d}$; 
ii) $\varphi_{\varepsilon}$ is of class $C^{1}\left(\mathbb{R}^{d} ; \mathbb{R}\right)$ with $\nabla \varphi_{\varepsilon}=\beta_{\varepsilon}$;

iii) the infimum defining $\varphi_{\varepsilon}(x)$ is attained at $\pi_{\varepsilon} x$ and

$$
\varphi_{\varepsilon}(x)=\frac{\varepsilon}{2}\left|\beta_{\varepsilon}(x)\right|^{2}+\varphi\left(\pi_{\varepsilon} x\right) ;
$$

iv) letting $\varepsilon \downarrow 0$, we have $\varphi_{\varepsilon}(x) \uparrow \varphi(x)$ for all $x \in \mathbb{R}^{d}$;

v) there exists $c>0$ such that for any $x \in \mathbb{R}^{d}$,

$$
-c(1+|x|) \leq \varphi\left(\pi_{\varepsilon} x\right) \leq \varphi_{\varepsilon}(x) \leq \varphi(x) .
$$

If $\varphi$ is the indicator of a convex nonempty subset $D$, then

$$
\begin{array}{rlrl}
\pi_{\varepsilon} x & =\operatorname{proj}_{D}(x) & & \forall \varepsilon>0, \forall x \in \mathbb{R}^{d} ; \\
\beta_{\varepsilon}(x) & =\frac{1}{\varepsilon}\left(x-\operatorname{proj}_{D}(x)\right) & \forall \varepsilon>0, \forall x \in \mathbb{R}^{d} .
\end{array}
$$

We conclude this subsection by stating some properties which will be needed in the sequel. The proof of the first proposition may be found in [8] and the proof of the second one is easy, hence it will be omitted.

Proposition 2.4 For any $a \in \mathbb{R}^{d}$, there exist constants $r>0, c>0$ (depending only on $\varphi$ ) such that, for each $\varepsilon \in \mathbb{R}^{*}, x \in \mathbb{R}^{d}$,

$$
\left\langle\beta_{\varepsilon}(x), x-a\right\rangle \geq r\left|\beta_{\varepsilon}(x)\right|+\varepsilon\left|\beta_{\varepsilon}(x)\right|^{2}-c(|x|+1) .
$$

Proposition 2.5 Let $F$ be a convex, continuous, differentiable function, from $\mathbb{R}^{d}$ into $\mathbb{R}$. Then the implicit Euler scheme with step size $\alpha$

$$
y_{n+1}=y_{n}+\alpha f\left(y_{n+1}\right), \quad 0 \leq n \leq N-1,
$$

where $f=-\nabla F$, is well-defined (i.e the equation $y-\alpha f(y)=c, c \in \mathbb{R}^{d}$ has a unique solution for all $\alpha>0)$. Moreover, the sequence $\left\{F\left(y_{n}\right)\right\}_{n \in \mathbb{N}}$ is decreasing.

\subsection{Multivalued stochastic differential equations}

Let be given

i) $d \in \mathbb{N}^{*}$ and $0<T<+\infty$;

ii) $\varphi: \mathbb{R}^{d} \rightarrow(-\infty ;+\infty]$ convex, l.s.c. and proper;

iii) $b: \mathbb{R}^{d} \rightarrow \mathbb{R}^{d}$ and $\sigma: \mathbb{R}^{d} \rightarrow \mathbb{R}^{d} \otimes \mathbb{R}^{d}$ Lipschitz continuous mappings;

iv) $(\Omega, \mathcal{F}, \mathbb{P})$ a probability space with filtration $\mathcal{F}_{t}$ satisfying the usual conditions;

v) $B=\left\{B_{t}, \mathcal{F}_{t} ; 0 \leq t \leq \infty\right\}$ a $d$-dimensional standard Brownian motion defined on $\left(\Omega, \mathcal{F}, \mathcal{F}_{t}, \mathbb{P}\right)$ with $B_{0}=0$ 
vi) $\xi$ a r.v. defined on $\left(\Omega, \mathcal{F}_{0}, \mathbb{P}\right)$, and taking values in $\overline{\operatorname{dom}(\varphi)} \mathbb{P}$-a.s.

With the previous data and assumptions, we shall approximate and simulate the unique solution $\left(X_{t}\right)_{0 \leq t \leq T}$ of the multivalued stochastic differential equation

$$
\left\{\begin{array}{l}
d X_{t}+\partial \varphi\left(X_{t}\right) d t \ni b\left(X_{t}\right) d t+\sigma\left(X_{t}\right) d B_{t} \\
X_{0}=\xi
\end{array}\right.
$$

By definition, a solution to the above equation is a pair of processes $(X, K)$ such that:

i) $X=\left\{X_{t}, 0 \leq t \leq T\right\}$ is a continuous, adapted process with values in $\overline{\operatorname{dom}(\varphi)}$ and $X_{0}=\xi$

ii) $K=\left\{K_{t}, 0 \leq t \leq T\right\}$ is a continuous, adapted process with bounded variation taking values in $\mathbb{R}^{d}$ with $K_{0}=0$;

iii) $d X_{t}=b\left(X_{t}\right) d t+\sigma\left(X_{t}\right) d B_{t}-d K_{t}$;

iv) for every pair of continuous, adapted process $(\alpha, \beta)$ taking values in $\mathbb{R}^{d}$ and satisfying

$$
\left(\alpha_{u}, \beta_{u}\right) \in \operatorname{Gr}(\partial \varphi) \quad \forall u \in[0, T]
$$

the measure $\left\langle X_{u}-\alpha_{u}, d K_{u}-\beta_{u} d u\right\rangle$ is $\mathbb{P}$-a.s. nonnegative on $[0, T]$.

We now give the plan of the paper. For each $\varepsilon>0$ fixed, we firstly give an approximate solution which is the solution of the ordinary stochastic differential equation

$$
\begin{cases}d X_{t}^{\varepsilon} & =b\left(X_{t}^{\varepsilon}\right) d t-\beta_{\varepsilon}\left(X_{t}^{\varepsilon}\right) d t+\sigma\left(X_{t}^{\varepsilon}\right) d B_{t} \\ X_{0}^{\varepsilon} & =X_{0}\end{cases}
$$

Next we estimate of the term $\mathbb{E}\left[\sup _{t \leq T}\left|X_{t}-X_{t}^{\varepsilon}\right|^{p}\right], p \geq 2$, to obtain the order of the approximation.

We secondly propose a semi-implicit Euler scheme with step size $\alpha=\frac{T}{N}, N \in \mathbb{N}^{*}$ on the time interval $[0, T]$ to approximate the solution $\left(X_{t}^{\varepsilon}\right)_{0 \leq t \leq T}$

$$
\left\{\begin{aligned}
\bar{X}_{0}^{\varepsilon} & =X_{0} \mathbb{P}-\text { p.s. } \\
\bar{X}_{(n+1) \alpha}^{\varepsilon} & =\bar{X}_{n \alpha}^{\varepsilon}+\alpha b\left(\bar{X}_{n \alpha}^{\varepsilon}\right)-\alpha \beta_{\varepsilon}\left(\bar{X}_{(n+1) \alpha}^{\varepsilon}\right)+\sigma\left(\bar{X}_{n \alpha}^{\varepsilon}\right)\left(B_{(n+1) \alpha}-B_{n \alpha}\right) \\
\bar{X}_{t}^{\varepsilon} & =\bar{X}_{n \alpha}^{\varepsilon} \quad \text { if } t \in[n \alpha,(n+1) \alpha)
\end{aligned}\right.
$$

and estimate the term $\mathbb{E}\left[\sup _{0 \leq t \leq T}\left|X_{t}^{\varepsilon}-\bar{X}_{t}^{\varepsilon}\right|^{p}\right], p \geq 2$. Putting together both approximations we obtain

$$
\mathbb{E}\left[\sup _{0 \leq t \leq T}\left|X_{t}-\bar{X}_{t}^{\varepsilon}\right|^{p}\right] \leq c(p) \varepsilon^{\frac{p}{8}}+c_{2}(p) \frac{\alpha^{\frac{p}{2}}}{\varepsilon^{p}} .
$$

Since the estimation is valid for all $p \geq 2$, we moreover get the pathwise convergence.

Before going to the main section, we note that if the gradient of the function $\varphi$ is repulsive enough, then there is no local time at the boundary of the domain. If moreover $\varphi$ belongs to 
$C^{1}(\operatorname{Int}(\operatorname{dom}(\varphi)))$, equation (9) turns into the ordinary stochastic differential equation

$$
\left\{\begin{array}{l}
d X_{t}=b\left(X_{t}\right) d t-\nabla \varphi\left(X_{t}\right) d t+\sigma\left(X_{t}\right) d B_{t} \\
X_{0}=\xi
\end{array}\right.
$$

with exploding drift at the boundary of the domain.

\section{Approximate solution}

Several authors have proved by the penalization method the convergence in $L^{2}$ of the sequence of the approximate solutions $X^{\varepsilon}$ to the unique solution $X$ of the multivalued stochastic differential equation (9) (see, e.g. [1], [8]).

In [21], the estimation

$$
\mathbb{E}\left[\sup _{0 \leq t \leq T}\left|X_{t}^{\varepsilon}-X_{t}\right|^{2}\right] \leq c \varepsilon^{\frac{1}{12}} \quad, \quad 0<T<+\infty
$$

was proved. We will generalize the result to the $L^{p}$-case $(p \geq 2)$ and find a larger power.

\subsection{Main estimates}

We firstly recall the main steps of Storm's proof in [21].

Proposition 3.1 Let $p \geq 2$ and assume $X_{0} \in L^{p}$. Then for every $\varepsilon>0$ there exists a constant $c$ independent of $\varepsilon$ such that

$$
\mathbb{E}\left[\sup _{t \leq T}\left|X_{t}^{\varepsilon}\right|^{p}+\left(\int_{0}^{T}\left|\beta_{\varepsilon}\left(X_{s}^{\varepsilon}\right)\right| d s\right)^{\frac{p}{2}}+\left(\int_{0}^{T} \varepsilon\left|\beta_{\varepsilon}\left(X_{s}^{\varepsilon}\right)\right|^{2} d s\right)^{\frac{p}{2}}\right] \leq c .
$$

Proposition 3.2 Let $p \geq 2$ and assume $X_{0} \in L^{2 p}$. Then for every $0<\varepsilon<1$ and $\delta>0$, we have

$$
\mathbb{E}\left[\sup _{t \leq T}\left|X_{t}^{\varepsilon}-X_{t}^{\delta}\right|^{p}\right] \leq c \mathbb{E}\left[\sup _{t \leq T}\left|X_{t}^{\varepsilon}-\pi_{\varepsilon} X_{t}^{\varepsilon}\right|^{p}\right]^{\frac{1}{2}}+c \mathbb{E}\left[\sup _{t \leq T}\left|X_{t}^{\delta}-\pi_{\delta} X_{t}^{\delta}\right|^{p}\right]^{\frac{1}{2}} .
$$

\subsection{Convergence rate}

Proposition 3.3 Let $p \geq 2$ and assume $X_{0} \in L^{5 p / 2}$ and $\mathbb{E}\left[\left|\varphi\left(X_{0}\right)\right|^{p}\right]<\infty$. Then for every $0<\varepsilon<1$ we have

$$
\mathbb{E}\left[\sup _{t \leq T}\left|X_{t}^{\varepsilon}-\pi_{\varepsilon} X_{t}^{\varepsilon}\right|^{2 p}\right] \leq c \varepsilon^{\frac{p}{2}} .
$$

Proof: Since $\varphi_{\varepsilon}$ is only $C^{1}$, we shall need the easy following lemma which can be proved by regularizing the function $f$ and using the Itô formula. 
Lemma 3.4 Let $f$ be a convex function of class $C^{1}\left(\mathbb{R}^{d} ; \mathbb{R}\right)$ whose gradient is Lipschitz continuous with constant $\gamma$. Then for all $p \geq 1$, we have almost surely

$$
\begin{aligned}
\left|f\left(X_{t \wedge \tau_{k}}^{\varepsilon}\right)\right|^{2 p} \leq & \left|f\left(X_{0}^{\varepsilon}\right)\right|^{2 p}+2 p \int_{0}^{t \wedge \tau_{k}}\left\langle\left|f\left(X_{s}^{\varepsilon}\right)\right|^{2 p-1} \nabla f\left(X_{s}^{\varepsilon}\right), \sigma\left(X_{s}^{\varepsilon}\right) d B_{s}\right\rangle \\
& +c \int_{0}^{t \wedge \tau_{k}}\left(\left|f\left(X_{s}^{\varepsilon}\right)\right|^{2 p-2}\left|\nabla f\left(X_{s}^{\varepsilon}\right)\right|^{2}+\gamma\left|f\left(X_{s}^{\varepsilon}\right)\right|^{2 p-1}\right)\left(\left|X_{s}^{\varepsilon}\right|+1\right)^{2} d s \\
& +c \int_{0}^{t \wedge \tau_{k}}\left|f\left(X_{s}^{\varepsilon}\right)\right|^{2 p-1}\left|\nabla f\left(X_{s}^{\varepsilon}\right)\right|\left(\left|X_{s}^{\varepsilon}\right|+1\right) d s \\
& -2 p \int_{0}^{t \wedge \tau_{k}}\left\langle\left|f\left(X_{s}^{\varepsilon}\right)\right|^{2 p-1} \nabla f\left(X_{s}^{\varepsilon}\right), \beta_{\varepsilon}\left(X_{s}^{\varepsilon}\right)\right\rangle d s .
\end{aligned}
$$

For every $k>0$, we define $\tau_{k}=\inf \left\{t:\left|X_{t}^{\varepsilon}\right| \geq k\right\}$. Applying the lemma to the convex function $\varphi_{\varepsilon}$ whose gradient is Lipschitz continuous with constant $\frac{1}{\varepsilon}$, taking the supremum over $t$ and taking expectations, we get

$$
\begin{aligned}
\mathbb{E}\left[\sup _{s \leq t \wedge \tau_{k}} \varphi_{\varepsilon}\left(X_{s}^{\varepsilon}\right)^{2 p}\right] \leq & \mathbb{E}\left[\varphi_{\varepsilon}\left(X_{0}^{\varepsilon}\right)^{2 p}\right]+\frac{c}{\varepsilon} \mathbb{E}\left[\int_{0}^{t \wedge \tau_{k}}\left|\varphi_{\varepsilon}\left(X_{s}^{\varepsilon}\right)\right|^{2 p-1}\left(\left|X_{s}^{\varepsilon}\right|+1\right)^{2} d s\right] \\
& +c \mathbb{E}\left[\int_{0}^{t \wedge \tau_{k}}\left|\varphi_{\varepsilon}\left(X_{s}^{\varepsilon}\right)\right|^{2 p-2}\left|\beta_{\varepsilon}\left(X_{s}^{\varepsilon}\right)\right|^{2}\left(\left|X_{s}^{\varepsilon}\right|+1\right)^{2} d s\right] \\
& +2 p \mathbb{E}\left[\sup _{s<t \wedge \tau_{k}}^{s}\left\langle\left|\varphi_{\varepsilon}\left(X_{u}^{\varepsilon}\right)\right|^{2 p-1} \beta_{\varepsilon}\left(X_{u}^{\varepsilon}\right), \sigma\left(X_{u}^{\varepsilon}\right) d B_{u}\right\rangle\right] \\
& +c \mathbb{E}\left[\int_{0}^{t \wedge \tau_{k}}\left|\varphi_{\varepsilon}\left(X_{s}^{\varepsilon}\right)\right|^{2 p-1}\left|\beta_{\varepsilon}\left(X_{s}^{\varepsilon}\right)\right|\left(\left|X_{s}^{\varepsilon}\right|+1\right) d s\right]
\end{aligned}
$$

Step 1: Estimate of the second term in the right hand side of (16).

The convex function $\varphi$ is bounded below by an affine function. Moreover $\pi_{\varepsilon}$ is a contraction and $\beta_{\varepsilon}(x) \in \partial \varphi\left(\pi_{\varepsilon} x\right)$. So we have, for every $x \in \mathbb{R}^{d}$,

$$
-c(1+|x|) \leq \varphi\left(\pi_{\varepsilon} x\right) \leq\left|\beta_{\varepsilon}(x)\right||x-a|+c .
$$

Hence from the definition of $\varphi_{\varepsilon}$ and Proposition 2.4, we have

$$
\left|\varphi_{\varepsilon}(x)\right|=\left.\left|\frac{\varepsilon}{2}\right| \beta_{\varepsilon}(x)\right|^{2}+\left.\varphi\left(\pi_{\varepsilon} x\right)\left|\leq \frac{\varepsilon}{2}\right| \beta_{\varepsilon}(x)\right|^{2}+c+c|x|+c(1+|x|)\left|\beta_{\varepsilon}(x)\right| .
$$

Applying this inequality to the second term on the right hand of (16), we obtain

$$
\begin{aligned}
\frac{c}{\varepsilon} \mathbb{E} & {\left[\int_{0}^{t \wedge \tau_{k}}\left|\varphi_{\varepsilon}\left(X_{s}^{\varepsilon}\right)\right|^{2 p-1}\left(\left|X_{s}^{\varepsilon}\right|+1\right)^{2} d s\right] } \\
\leq & c \varepsilon^{-2 p+1} \mathbb{E}\left[\sup _{s \leq t \wedge \tau_{k}} \varepsilon^{2 p-2}\left|\varphi_{\varepsilon}\left(X_{s}^{\varepsilon}\right)\right|^{2 p-2}\left(1+\sup _{s \leq t \wedge \tau_{k}}\left|X_{s}^{\varepsilon}\right|\right)^{2} \int_{0}^{t \wedge \tau_{k}} \varepsilon\left|\beta_{\varepsilon}\left(X_{s}^{\varepsilon}\right)\right|^{2} \mid d s\right] \\
& +c \varepsilon^{-2 p+1} t \mathbb{E}\left[\sup _{s \leq t \wedge \tau_{k}} \varepsilon^{2 p-2}\left|\varphi_{\varepsilon}\left(X_{s}^{\varepsilon}\right)\right|^{2 p-2}\left(1+\sup _{s \leq t \wedge \tau_{k}}\left|X_{s}^{\varepsilon}\right|\right)^{3}\right] \\
& +c \varepsilon^{-2 p+1} \mathbb{E}\left[\sup _{s \leq t \wedge \tau_{k}} \varepsilon^{2 p-2}\left|\varphi_{\varepsilon}\left(X_{s}^{\varepsilon}\right)\right|^{2 p-2}\left(1+\sup _{s \leq t \wedge \tau_{k}}\left|X_{s}^{\varepsilon}\right|\right)^{3} \int_{0}^{t \wedge \tau_{k}}\left|\beta_{\varepsilon}\left(X_{s}^{\varepsilon}\right)\right| d s\right]
\end{aligned}
$$


Using the Hölder inequality, we get

$$
\begin{aligned}
& \frac{c}{\varepsilon} \mathbb{E}\left[\int_{0}^{t \wedge \tau_{k}}\left|\varphi_{\varepsilon}\left(X_{s}^{\varepsilon}\right)\right|^{2 p-1}\left(\left|X_{s}^{\varepsilon}\right|+1\right)^{2} d s\right] \\
& \leq c \varepsilon^{-2 p+1} \mathbb{E}\left[\sup _{s \leq t \wedge \tau_{k}} \varepsilon^{2 p}\left|\varphi_{\varepsilon}\left(X_{s}^{\varepsilon}\right)\right|^{2 p}\right]^{\frac{2 p-2}{2 p}} \mathbb{E}\left[\left(\int_{0}^{t \wedge \tau_{k}} \varepsilon\left|\beta_{\varepsilon}\left(X_{s}^{\varepsilon}\right)\right|^{2} d s\right)^{2 p}+\left(1+\sup _{s \leq t \wedge \tau_{k}}\left|X_{s}^{\varepsilon}\right|\right)^{4 p}\right]^{\frac{1}{p}} \\
& +c \varepsilon^{-2 p+1} t \mathbb{E}\left[\sup _{s \leq t \wedge \tau_{k}} \varepsilon^{2 p}\left|\varphi_{\varepsilon}\left(X_{s}^{\varepsilon}\right)\right|^{2 p}\right]^{\frac{2 p-2}{2 p}} \mathbb{E}\left[\left(1+\sup _{s \leq t \wedge \tau_{k}}\left|X_{s}^{\varepsilon}\right|\right)^{3 p}\right]^{\frac{1}{p}} \\
& +c \varepsilon^{-2 p+1} \mathbb{E}\left[\sup _{s \leq t \wedge \tau_{k}} \varepsilon^{2 p}\left|\varphi_{\varepsilon}\left(X_{s}^{\varepsilon}\right)\right|^{2 p}\right]^{\frac{p-1}{p}} \mathbb{E}\left[\left(1+\sup _{s \leq t \wedge \tau_{k}}\left|X_{s}^{\varepsilon}\right|\right)^{5 p}\right]^{\frac{3}{5 p}} \mathbb{E}\left[\left(\int_{0}^{t \wedge \tau_{k}}\left|\beta_{\varepsilon}\left(X_{s}^{\varepsilon}\right)\right| d s\right)^{\frac{5 p}{2}}\right]^{\frac{2}{5 p}}
\end{aligned}
$$

Hence if $X_{0} \in L^{5 p}$, by Proposition 3.1, we have

$$
\frac{c}{\varepsilon} \mathbb{E}\left[\int_{0}^{t \wedge \tau_{k}}\left|\varphi_{\varepsilon}\left(X_{s}^{\varepsilon}\right)\right|^{2 p-1}\left(\left|X_{s}^{\varepsilon}\right|+1\right)^{2} d s\right] \leq c \varepsilon^{-2 p+1} \mathbb{E}\left[\sup _{s \leq t \wedge \tau_{k}} \varepsilon^{2 p}\left|\varphi_{\varepsilon}\left(X_{s}^{\varepsilon}\right)\right|^{2 p}\right]^{\frac{2 p-2}{2 p}} .
$$

Step 2: Estimate of the third term on the right hand side of (16).

If $X_{0} \in L^{4 p}$, by the Hölder inequality and Proposition 3.1, we have

$$
c \mathbb{E}\left[\int_{0}^{t \wedge \tau_{k}}\left|\varphi_{\varepsilon}\left(X_{s}^{\varepsilon}\right)\right|^{2 p-2}\left|\beta_{\varepsilon}\left(X_{s}^{\varepsilon}\right)\right|^{2}\left(\left|X_{s}^{\varepsilon}\right|+1\right)^{2} d s\right] \leq c \varepsilon^{-2 p+1} \mathbb{E}\left[\sup _{s \leq t \wedge \tau_{k}} \varepsilon^{2 p}\left|\varphi_{\varepsilon}\left(X_{s}^{\varepsilon}\right)\right|^{2 p}\right]^{\frac{p-1}{p}}
$$

Step 3: Estimate of the fourth term on the right hand side of (16).

Using Doob's maximal inequality, we get

$$
\begin{aligned}
& 2 p \mathbb{E}\left[\sup _{s \leq t \wedge \tau_{k}} \int_{0}^{s}\left\langle\left|\varphi_{\varepsilon}\left(X_{u}^{\varepsilon}\right)\right|^{2 p-1} \beta_{\varepsilon}\left(X_{u}^{\varepsilon}\right), \sigma\left(X_{u}^{\varepsilon}\right) d B_{u}\right\rangle\right] \\
& \leq c \varepsilon^{-\frac{4 p-1}{2}} \mathbb{E}\left[\sup _{s \leq t \wedge \tau_{k}} \varepsilon^{2 p-1}\left|\varphi_{\varepsilon}\left(X_{s}^{\varepsilon}\right)\right|^{2 p-1}\left(\int_{0}^{t \wedge \tau_{k}} \varepsilon\left|\beta_{\varepsilon}\left(X_{s}^{\varepsilon}\right)\right|^{2}\left(\left|X_{s}^{\varepsilon}\right|+1\right)^{2} d s\right)^{\frac{1}{2}}\right] .
\end{aligned}
$$

If $X_{0} \in L^{4 p}$ then, by the Hölder inequality and Proposition 3.1, we obtain

$$
2 p \mathbb{E}\left[\sup _{s \leq t \wedge \tau_{k}} \int_{0}^{s}\left\langle\left|\varphi_{\varepsilon}\left(X_{u}^{\varepsilon}\right)\right|^{2 p-1} \beta_{\varepsilon}\left(X_{u}^{\varepsilon}\right), \sigma\left(X_{u}^{\varepsilon}\right) d B_{u}\right\rangle\right] \leq c \varepsilon^{-\frac{4 p-1}{2}} \mathbb{E}\left[\sup _{s \leq t \wedge \tau_{k}} \varepsilon^{2 p}\left|\varphi_{\varepsilon}\left(X_{s}^{\varepsilon}\right)\right|^{2 p}\right]^{\frac{2 p-1}{2 p}}
$$

Step 4: Estimate of the fifth term on the right hand side of (16).

Since

$$
\begin{aligned}
& c \mathbb{E}\left[\int_{0}^{t \wedge \tau_{k}}\left|\varphi_{\varepsilon}\left(X_{s}^{\varepsilon}\right)\right|^{2 p-1}\left|\beta_{\varepsilon}\left(X_{s}^{\varepsilon}\right)\right|\left(\left|X_{s}^{\varepsilon}\right|+1\right) d s\right] \\
& \leq \quad c \varepsilon^{\frac{-4 p+1}{2}} \mathbb{E}\left[\sup _{s \leq t \wedge \tau_{k}} \varepsilon^{2 p-1}\left|\varphi_{\varepsilon}\left(X_{s}^{\varepsilon}\right)\right|^{2 p-1} \int_{0}^{t \wedge \tau_{k}} \varepsilon^{\frac{1}{2}}\left|\beta_{\varepsilon}\left(X_{s}^{\varepsilon}\right)\right|\left(\left|X_{s}^{\varepsilon}\right|+1\right) d s\right] .
\end{aligned}
$$


Using the Cauchy and Hölder inequalities, and Proposition 3.1, if $X_{0} \in L^{4 p}$, we have

$$
c \mathbb{E}\left[\int_{0}^{t \wedge \tau_{k}}\left|\varphi_{\varepsilon}\left(X_{s}^{\varepsilon}\right)\right|^{2 p-1}\left|\beta_{\varepsilon}\left(X_{s}^{\varepsilon}\right)\right|\left(\left|X_{s}^{\varepsilon}\right|+1\right) d s\right] \leq c \varepsilon^{\frac{-4 p+1}{2}} \mathbb{E}\left[\sup _{s \leq t \wedge \tau_{k}} \varepsilon^{2 p}\left|\varphi_{\varepsilon}\left(X_{s}^{\varepsilon}\right)\right|^{2 p}\right]^{\frac{2 p-1}{2 p}}
$$

By (17)-(20), (16) becomes

$$
\begin{aligned}
\mathbb{E}\left[\varepsilon^{2 p}\left|\varphi_{\varepsilon}\left(X_{t \wedge \tau_{k}}^{\varepsilon}\right)\right|^{2 p}\right] \leq \varepsilon^{2 p} \mathbb{E}\left[\left|\varphi_{\varepsilon}\left(X_{0}\right)\right|^{2 p}\right] & +c \varepsilon^{\frac{1}{2}} \mathbb{E}\left[\sup _{s \leq t \wedge \tau_{k}} \varepsilon^{2 p}\left|\varphi_{\varepsilon}\left(X_{s}^{\varepsilon}\right)\right|^{2 p}\right]^{\frac{2 p-1}{2 p}} \\
& +c \varepsilon \mathbb{E}\left[\sup _{s \leq t \wedge \tau_{k}} \varepsilon^{2 p}\left|\varphi_{\varepsilon}\left(X_{s}^{\varepsilon}\right)\right|^{2 p}\right]^{\frac{p-1}{p}}
\end{aligned}
$$

We define $A=\varepsilon^{-p} \mathbb{E}\left[\sup _{s \leq t \wedge \tau_{k}} \varepsilon^{2 p}\left|\varphi_{\varepsilon}\left(X_{s}^{\varepsilon}\right)\right|^{2 p}\right]$, then $A<\infty$.

Since $-c(1+|x|) \leq \varphi_{\varepsilon}(x) \leq \varphi(x)$ and $\mathbb{E}\left[\left|\varphi\left(X_{0}\right)\right|^{2 p}\right] \leq c,(21)$ yields

$$
\frac{A}{c\left(\varepsilon^{p}+A^{1-\frac{1}{2 p}}+A^{1-\frac{1}{p}}\right)} \leq 1
$$

As $\varepsilon<1$, we see that $A$ is uniformly bounded (with respect to $\varepsilon$ and $k$ ) by some constant. After delocalization, it follows that for any $p \geq 1$, if $X_{0} \in L^{5 p}$ and $\mathbb{E}\left[\left|\varphi\left(X_{0}\right)\right|^{2 p}\right]<\infty$,

$$
\mathbb{E}\left[\sup _{t \leq T} \varepsilon^{2 p}\left|\varphi_{\varepsilon}\left(X_{t}^{\varepsilon}\right)\right|^{2 p}\right] \leq c \varepsilon^{p}
$$

Now using the bounds on $\varphi\left(\pi_{\varepsilon} x\right)$ given by (6), we obtain

$$
\left|\varphi\left(\pi_{\varepsilon} x\right)\right|^{2 p} \leq c\left(1+|x|^{2 p}+\left|\varphi_{\varepsilon}(x)\right|^{2 p}\right) .
$$

From inequality (23), we deduce

$$
\mathbb{E}\left[\sup _{t \leq T} \varepsilon^{2 p}\left|\varphi\left(\pi_{\varepsilon} X_{t}^{\varepsilon}\right)\right|^{2 p}\right] \leq c \varepsilon^{p}
$$

Using the inequality $c a^{2 p}-b^{2 p} \leq(a+b)^{2 p}$ and (23)-(24), we have

$$
\begin{aligned}
\mathbb{E}\left[\sup _{t \leq T}\left|X_{t}^{\varepsilon}-\pi_{\varepsilon} X_{t}^{\varepsilon}\right|^{4 p}\right] & \leq c \mathbb{E}\left[\sup _{t \leq T}|| X_{t}^{\varepsilon}-\left.\pi_{\varepsilon} X_{t}^{\varepsilon}\right|^{2}+\left.2 \varepsilon \varphi\left(\pi_{\varepsilon} X_{t}^{\varepsilon}\right)\right|^{2 p}\right] \\
& +c \mathbb{E}\left[\sup _{t \leq T} \varepsilon^{2 p}\left|\varphi\left(\pi_{\varepsilon} X_{t}^{\varepsilon}\right)\right|^{2 p}\right] \\
& \leq c \mathbb{E}\left[\sup _{t \leq T} \varepsilon^{2 p}\left|\varphi_{\varepsilon}\left(X_{t}^{\varepsilon}\right)\right|^{2 p}\right]+c \mathbb{E}\left[\sup _{t \leq T} \varepsilon^{2 p}\left|\varphi\left(\pi_{\varepsilon} X_{t}^{\varepsilon}\right)\right|^{2 p}\right] \\
& \leq c \varepsilon^{p}
\end{aligned}
$$


which completes the proof of the proposition.

Thanks to Proposition 3.2, for every $\varepsilon>0$ and $\delta>0$, we have

$$
\mathbb{E}\left[\sup _{t \leq T}\left|X_{t}^{\varepsilon}-X_{t}^{\delta}\right|^{2 p}\right] \leq c\left(\varepsilon^{\frac{p}{4}}+\delta^{\frac{p}{4}}\right) .
$$

We have proved that the sequence of process $\left(X_{t}^{\varepsilon}\right)_{0 \leq t \leq T}$ is Cauchy in $L^{p}$, and so converges to a continuous process $\left(X_{t}\right)_{0 \leq t \leq T}$. By the end of the proof of Theorem 3.2 in [21], we can show that the limit is the unique solution of the multivalued stochastic differential equation (9) for which we have

$$
\mathbb{E}\left[\sup _{t \leq T}\left|X_{t}-X_{t}^{\varepsilon}\right|^{p}\right] \leq c \varepsilon^{\frac{p}{8}} \quad \forall p \geq 2
$$

Taking $\varepsilon=1 / n$, we may obtain the a.s. uniform convergence for $p>8$.

\section{Euler semi-implicit scheme}

The book [15] is a good reference for the discretized simulation of ordinary stochastic differential equations with Lipschitz coefficients. We are interested here in the case where the coefficients are more singular. On other words, the diffusion coefficient remains Lipschitz continuous but the drift one is the sum of a Lipschitz term and a monotone one.

\subsection{Euler semi-implicit scheme}

Let $p \geq 2,0<T<\infty$ and $N \in \mathbb{N}^{*}$. We propose a Euler semi-implicit scheme with step size $\alpha=\frac{\bar{T}}{N}$ associated to the process $\left(X_{t}\right)_{0 \leq t \leq T}$ :

$$
\begin{cases}\bar{X}_{0}^{\varepsilon} & =x \in \operatorname{Int}(\operatorname{dom}(\varphi)) \\ \bar{X}_{(n+1) \alpha}^{\varepsilon} & =\bar{X}_{n \alpha}^{\varepsilon}+\alpha b\left(\bar{X}_{n \alpha}^{\varepsilon}\right)-\alpha \beta_{\varepsilon}\left(\bar{X}_{(n+1) \alpha}^{\varepsilon}\right)+\sigma\left(\bar{X}_{n \alpha}^{\varepsilon}\right)\left(B_{(n+1) \alpha}-B_{n \alpha}\right) .\end{cases}
$$

By Proposition (2.5), it is easy to see that this scheme is well defined. We also introduce the continuity version: if $t \in[n \alpha,(n+1) \alpha)$, then

$$
\bar{X}_{t}^{\varepsilon}=\bar{X}_{n \alpha}^{\varepsilon}+(t-n \alpha) b\left(\bar{X}_{n \alpha}^{\varepsilon}\right)-(t-n \alpha) \beta_{\varepsilon}\left(\bar{X}_{(n+1) \alpha}^{\varepsilon}\right)+\sigma\left(\bar{X}_{n \alpha}^{\varepsilon}\right)\left(B_{t}-B_{n \alpha}\right) .
$$

We first state an simple elementary inequality which looks like a discrete Itô formula.

Lemma 4.1 Let $\left\{c_{k}\right\}_{k \in \mathbb{N}}$ and $\left\{d_{k}\right\}_{k \in \mathbb{N}}$ two sequences of $\mathbb{R}^{d}$. For every $n \in \mathbb{N}^{*}$, we have

$$
\left|\sum_{k=0}^{n}\left(c_{k}+d_{k}\right)\right|^{2} \leq \sum_{k=0}^{n}\left|c_{k}\right|^{2}+\sum_{k=0}^{n}\left\langle 2 \sum_{i=0}^{k-1}\left(c_{i}+d_{i}\right), c_{k}\right\rangle+2 \sum_{k=0}^{n}\left\langle\sum_{i=0}^{k}\left(c_{i}+d_{i}\right), d_{k}\right\rangle .
$$




\subsection{Estimate in $L^{p}$}

We know from the previous section that any random variable $X_{t}^{\varepsilon}$ belongs to $L^{p}$. This is also true for the discretized process.

Proposition 4.2 There exists a positive $c$ independent of $\varepsilon$ and $\alpha$ such that

$$
\mathbb{E}\left[\sup _{n \leq N}\left|\bar{X}_{n \alpha}^{\varepsilon}\right|^{p}\right] \leq c .
$$

Proof: By formula (26), we can write

$$
\bar{X}_{n \alpha}^{\varepsilon}-x=\sum_{k=0}^{n-1}\left(\alpha b\left(\bar{X}_{k \alpha}^{\varepsilon}\right)+\sigma\left(\bar{X}_{k \alpha}^{\varepsilon}\right)\left(B_{(k+1) \alpha}-B_{k \alpha}\right)\right)-\sum_{k=0}^{n-1} \alpha \beta_{\varepsilon}\left(\bar{X}_{(k+1) \alpha}^{\varepsilon}\right) .
$$

Applying Lemma 4.1, we get

$$
\begin{aligned}
\left|\bar{X}_{n \alpha}^{\varepsilon}-x\right|^{2} \leq & \sum_{k=0}^{n-1}\left|\alpha b\left(\bar{X}_{k \alpha}^{\varepsilon}\right)+\sigma\left(\bar{X}_{k \alpha}^{\varepsilon}\right)\left(B_{(k+1) \alpha}-B_{k \alpha}\right)\right|^{2} \\
& +2 \sum_{k=0}^{n-1}\left\langle\bar{X}_{k \alpha}^{\varepsilon}-x, \alpha b\left(\bar{X}_{k \alpha}^{\varepsilon}\right)+\sigma\left(\bar{X}_{k \alpha}^{\varepsilon}\right)\left(B_{(k+1) \alpha}-B_{k \alpha}\right)\right\rangle \\
& -2 \sum_{k=0}^{n-1}\left\langle\bar{X}_{(k+1) \alpha}^{\varepsilon}-x, \alpha \beta_{\varepsilon}\left(\bar{X}_{(k+1) \alpha}^{\varepsilon}\right)\right\rangle .
\end{aligned}
$$

Since $x \in \operatorname{Int}(\operatorname{dom}(\varphi))$, the proposition 2.4 is valid, and by definition de $\bar{X}_{(k+1) \alpha}^{\varepsilon}$, we obtain

$$
\begin{aligned}
-\left\langle\bar{X}_{(k+1) \alpha}^{\varepsilon}-x, \beta_{\varepsilon}\left(\bar{X}_{(k+1) \alpha}^{\varepsilon}\right)\right\rangle \leq & (c \alpha-r)\left|\beta_{\varepsilon}\left(\bar{X}_{(k+1) \alpha}^{\varepsilon}\right)\right|+c+c\left|\bar{X}_{k \alpha}^{\varepsilon}-x\right| \\
& +\alpha\left|b\left(\bar{X}_{k \alpha}^{\varepsilon}\right)-b(x)\right|+c\left|\sigma\left(\bar{X}_{k \alpha}^{\varepsilon}\right)\left(B_{(k+1) \alpha}-B_{k \alpha}\right)\right| .
\end{aligned}
$$

We can choose $\alpha$ small enough so that $\alpha \leq \frac{r}{c}$. By the Cauchy inequality and the Lipschitz property of $b,(30)$ becomes

$$
\begin{aligned}
\left|\bar{X}_{n \alpha}^{\varepsilon}-x\right|^{2} \leq c & \sum_{k=0}^{n-1} \alpha\left|\bar{X}_{k \alpha}^{\varepsilon}-x\right|^{2}+2 \sum_{k=0}^{n-1}\left|\sigma\left(\bar{X}_{k \alpha}^{\varepsilon}\right)\left(B_{(k+1) \alpha}-B_{k \alpha}\right)\right|^{2} \\
& +\sum_{k=0}^{n-1} \alpha\left|\sigma\left(\bar{X}_{k \alpha}^{\varepsilon}\right)\left(B_{(k+1) \alpha}-B_{k \alpha}\right)\right|+c \\
& +\sum_{k=0}^{n-1}\left\langle\bar{X}_{k \alpha}^{\varepsilon}-x, \sigma\left(\bar{X}_{k \alpha}^{\varepsilon}\right)\left(B_{(k+1) \alpha}-B_{k \alpha}\right)\right\rangle .
\end{aligned}
$$

Applying the following inequality

$$
\sum_{i=1}^{n}\left|x_{i}\right|^{m} \leq\left(\sum_{i=1}^{n}\left|x_{i}\right|\right)^{m} \leq n^{m-1} \sum_{i=1}^{n}\left|x_{i}\right|^{m} \quad \forall m \geq 1,
$$

and taking the expectation of supremum, we have 


$$
\begin{aligned}
& \mathbb{E}\left[\sup _{n \leq N}\left|\bar{X}_{n \alpha}^{\varepsilon}-x\right|^{p}\right] \leq c \mathbb{E}\left[\sup _{n \leq N}\left(\sum_{k=0}^{n-1} \alpha\left|\bar{X}_{k \alpha}^{\varepsilon}-x\right|^{2}\right)^{\frac{p}{2}}\right] \\
&+c \mathbb{E}\left[\sup _{n \leq N}\left(\sum_{k=0}^{n-1}\left|\sigma\left(\bar{X}_{k \alpha}^{\varepsilon}\right)\left(B_{(k+1) \alpha}-B_{k \alpha}\right)\right|^{2}\right)^{\frac{p}{2}}\right] \\
&+c \mathbb{E}\left[\sup _{n \leq N}\left(\sum_{k=0}^{n-1} \alpha\left|\sigma\left(\bar{X}_{k \alpha}^{\varepsilon}\right)\left(B_{(k+1) \alpha}-B_{k \alpha}\right)\right|\right)^{\frac{p}{2}}\right]+c \\
&+c \mathbb{E}\left[\sup _{n \leq N}\left(\sum_{k=0}^{n-1}\left\langle\bar{X}_{k \alpha}^{\varepsilon}-x, \sigma\left(\bar{X}_{k \alpha}^{\varepsilon}\right)\left(B_{(k+1) \alpha}-B_{k \alpha}\right)\right\rangle\right)^{\frac{p}{2}}\right] .
\end{aligned}
$$

Applying the Cauchy and Burkholder-Davis-Gundy inequalities to the two last terms on the right hand side, (32) becomes

$$
\begin{aligned}
\mathbb{E}\left[\sup _{n \leq N}\left|\bar{X}_{n \alpha}^{\varepsilon}-x\right|^{p}\right] \leq \frac{1}{2} \mathbb{E}\left[\sup _{n \leq N-1}\left|\bar{X}_{n \alpha}^{\varepsilon}-x\right|^{p}\right]+c \\
+c \mathbb{E}\left[\left(\sum_{k=0}^{N-1}\left|\sigma\left(\bar{X}_{k \alpha}^{\varepsilon}\right)\left(B_{(k+1) \alpha}-B_{k \alpha}\right)\right|^{2}\right)^{\frac{p}{2}}\right]
\end{aligned}
$$

Using again the inequality (31), we obtain

$$
\begin{aligned}
\mathbb{E}\left[\sup _{n \leq N}\left|\bar{X}_{n \alpha}^{\varepsilon}-x\right|^{p}\right] & \leq \frac{1}{2} \mathbb{E}\left[\sup _{n \leq N-1}\left|\bar{X}_{n \alpha}^{\varepsilon}-x\right|^{p}\right]+c \\
& +c N^{\frac{p}{2}-1} \sum_{n=0}^{N-1} \mathbb{E}\left[\left|\sigma\left(\bar{X}_{k \alpha}^{\varepsilon}\right)\left(B_{(k+1) \alpha}-B_{k \alpha}\right)\right|^{p}\right] \\
& \leq \frac{1}{2} \mathbb{E}\left[\sup _{n \leq N-1}\left|\bar{X}_{n \alpha}^{\varepsilon}-x\right|^{p}\right]+c \sum_{n=0}^{N-1} \alpha \mathbb{E}\left[\sup _{k \leq n}\left|\bar{X}_{k \alpha}^{\varepsilon}-x\right|^{p}\right]+c .
\end{aligned}
$$

After the usual delocalization and application of Gronwall inequality, we finally obtain

$$
\mathbb{E}\left[\sup _{n \leq N}\left|\bar{X}_{n \alpha}^{\varepsilon}-x\right|^{p}\right] \leq c
$$

which completes the proof of the proposition.

\subsection{Order of the Euler semi-implicit scheme}

Theorem 4.3 For every $p \geq 2$, there exists a positive $c(p)$ independent of $\alpha$ and $\varepsilon$ such that

$$
\mathbb{E}\left[\sup _{n \leq N}\left|X_{n \alpha}^{\varepsilon}-\bar{X}_{n \alpha}^{\varepsilon}\right|^{p}\right] \leq c(p) \frac{\alpha^{\frac{p}{2}}}{\varepsilon^{p}} .
$$


Proof: For every $1 \leq n \leq N$, we write $X_{n \alpha}^{\varepsilon}$ and $\bar{X}_{n \alpha}^{\varepsilon}$ with the form (29). Using Lemma 4.1, we have

$$
\begin{aligned}
\left|X_{n \alpha}^{\varepsilon}-\bar{X}_{n \alpha}^{\varepsilon}\right|^{2} \leq & 2 \sum_{k=0}^{n-1}\left|\int_{k \alpha}^{(k+1) \alpha}\left(b\left(X_{s}^{\varepsilon}\right)-b\left(\bar{X}_{k \alpha}^{\varepsilon}\right)\right) d s\right|^{2} \\
& +2 \sum_{k=0}^{n-1}\left|\int_{k \alpha}^{(k+1) \alpha}\left(\sigma\left(X_{s}^{\varepsilon}\right)-\sigma\left(\bar{X}_{k \alpha}^{\varepsilon}\right)\right) d B_{s}\right|^{2} \\
& +2 \sum_{k=0}^{n-1}\left\langle X_{k \alpha}^{\varepsilon}-\bar{X}_{k \alpha}^{\varepsilon}, \int_{k \alpha}^{(k+1) \alpha}\left(b\left(X_{s}^{\varepsilon}\right)-b\left(\bar{X}_{k \alpha}^{\varepsilon}\right)\right) d s\right\rangle \\
& +2 \sum_{k=0}^{n-1}\left\langle X_{k \alpha}^{\varepsilon}-\bar{X}_{k \alpha}^{\varepsilon}, \int_{k \alpha}^{(k+1) \alpha}\left(\sigma\left(X_{s}^{\varepsilon}\right)-\sigma\left(\bar{X}_{k \alpha}^{\varepsilon}\right)\right) d B_{s}\right\rangle \\
& -2 \sum_{k=0}^{n-1}\left\langle X_{(k+1) \alpha}^{\varepsilon}-\bar{X}_{(k+1) \alpha}^{\varepsilon}, \int_{k \alpha}^{(k+1) \alpha}\left(\beta_{\varepsilon}\left(X_{s}^{\varepsilon}\right)-\beta_{\varepsilon}\left(\bar{X}_{(k+1) \alpha}^{\varepsilon}\right)\right) d s\right\rangle
\end{aligned}
$$

By the monotony and the Lipschitz property with constant $\frac{1}{\varepsilon}$ of $\beta_{\varepsilon}$, we have

$$
\begin{aligned}
& -\left\langle X_{(k+1) \alpha}^{\varepsilon}-\bar{X}_{(k+1) \alpha}^{\varepsilon}, \int_{k \alpha}^{(k+1) \alpha}\left(\beta_{\varepsilon}\left(X_{s}^{\varepsilon}\right)-\beta_{\varepsilon}\left(\bar{X}_{(k+1) \alpha}^{\varepsilon}\right)\right) d s\right\rangle \\
& \leq \frac{\alpha}{2}\left|X_{(k+1) \alpha}^{\varepsilon}-\bar{X}_{(k+1) \alpha}^{\varepsilon}\right|^{2}+\frac{\alpha}{2 \varepsilon^{2}} \sup _{k \alpha \leq s \leq(k+1) \alpha}\left|X_{s}^{\varepsilon}-X_{(k+1) \alpha}^{\varepsilon}\right|^{2} .
\end{aligned}
$$

By the Lipschitz property of $b$ and the Cauchy inequality, (34) becomes

$$
\begin{aligned}
\left|X_{n \alpha}^{\varepsilon}-\bar{X}_{n \alpha}^{\varepsilon}\right|^{2} \leq & \alpha\left|X_{n \alpha}^{\varepsilon}-\bar{X}_{n \alpha}^{\varepsilon}\right|^{2}+c \sum_{k=0}^{n-1} \alpha\left|X_{k \alpha}^{\varepsilon}-\bar{X}_{k \alpha}^{\varepsilon}\right|^{2} \\
& +c \sum_{k=0}^{n-1}\left(\alpha \sup _{k \alpha \leq s \leq(k+1) \alpha}\left|X_{s}^{\varepsilon}-X_{k \alpha}^{\varepsilon}\right|^{2}+\frac{\alpha}{\varepsilon^{2}} \sup _{k \alpha \leq s \leq(k+1) \alpha}\left|X_{s}^{\varepsilon}-X_{(k+1) \alpha}^{\varepsilon}\right|^{2}\right) \\
& +2 \sum_{k=0}^{n-1}\left|\int_{k \alpha}^{(k+1) \alpha}\left(\sigma\left(X_{s}^{\varepsilon}\right)-\sigma\left(\bar{X}_{k \alpha}^{\varepsilon}\right)\right) d B_{s}\right|^{2} \\
& +2 \sum_{k=0}^{n-1}\left\langle X_{k \alpha}^{\varepsilon}-\bar{X}_{k \alpha}^{\varepsilon}, \int_{k \alpha}^{(k+1) \alpha}\left(\sigma\left(X_{s}^{\varepsilon}\right)-\sigma\left(\bar{X}_{k \alpha}^{\varepsilon}\right)\right) d B_{s}\right\rangle
\end{aligned}
$$

For any small enough $\alpha$, using the inequality (31) with power $\frac{p}{2}$ and taking the expectation of supremum, we obtain 


$$
\begin{aligned}
& \mathbb{E}\left[\sup _{n \leq N}\left|X_{n \alpha}^{\varepsilon}-\bar{X}_{n \alpha}^{\varepsilon}\right|^{p}\right] \\
& \leq c \mathbb{E}\left[\left(\sum_{k=0}^{N-1} \alpha\left|X_{k \alpha}^{\varepsilon}-\bar{X}_{k \alpha}^{\varepsilon}\right|^{2}\right)^{\frac{p}{2}}\right]+c \mathbb{E}\left[\left(\sum_{k=0}^{N-1}\left|\int_{k \alpha}^{(k+1) \alpha}\left(\sigma\left(X_{s}^{\varepsilon}\right)-\sigma\left(\bar{X}_{k \alpha}^{\varepsilon}\right)\right) d B_{s}\right|^{2}\right)^{\frac{p}{2}}\right] \\
& +c \mathbb{E}\left[\left(\sum_{k=0}^{N-1}\left(\alpha \sup _{k \alpha \leq s \leq(k+1) \alpha}\left|X_{s}^{\varepsilon}-X_{k \alpha}^{\varepsilon}\right|^{2}+\frac{\alpha}{\varepsilon^{2}} \sup _{k \alpha \leq s \leq(k+1) \alpha}\left|X_{s}^{\varepsilon}-X_{(k+1) \alpha}^{\varepsilon}\right|^{2}\right)\right)^{\frac{p}{2}}\right] \\
& +c \mathbb{E}\left[\sup _{n \leq N}\left|\sum_{k=0}^{n-1}\left\langle X_{k \alpha}^{\varepsilon}-\bar{X}_{k \alpha}^{\varepsilon}, \int_{k \alpha}^{(k+1) \alpha}\left(\sigma\left(X_{s}^{\varepsilon}\right)-\sigma\left(\bar{X}_{k \alpha}^{\varepsilon}\right)\right) d B_{s}\right\rangle\right|^{\frac{p}{2}}\right] .
\end{aligned}
$$

Applying (31) to each term on the right hand side, we have

$$
\begin{aligned}
& \mathbb{E}\left[\sup _{n \leq N}\left|X_{n \alpha}^{\varepsilon}-\bar{X}_{n \alpha}^{\varepsilon}\right|^{p}\right] \\
& \leq c N^{\frac{p}{2}-1} \sum_{k=0}^{N-1} \alpha^{\frac{p}{2}} \mathbb{E}\left[\left|X_{k \alpha}^{\varepsilon}-\bar{X}_{k \alpha}^{\varepsilon}\right|^{p}\right]+c N^{\frac{p}{2}-1} \sum_{k=0}^{N-1} \mathbb{E}\left[\left|\int_{k \alpha}^{(k+1) \alpha}\left(\sigma\left(X_{s}^{\varepsilon}\right)-\sigma\left(\bar{X}_{k \alpha}^{\varepsilon}\right)\right) d B_{s}\right|^{p}\right] \\
& +\frac{c}{\varepsilon^{p}} N^{\frac{p}{2}-1} \sum_{k=0}^{N-1} \alpha^{\frac{p}{2}}\left(\mathbb{E}\left[\sup _{k \alpha \leq s \leq(k+1) \alpha}\left|X_{s}^{\varepsilon}-X_{k \alpha}^{\varepsilon}\right|^{p}\right]+\mathbb{E}\left[\sup _{k \alpha \leq s \leq(k+1) \alpha}\left|X_{s}^{\varepsilon}-X_{(k+1) \alpha}^{\varepsilon}\right|^{p}\right]\right) d s \\
& \left.+c \mathbb{E}\left[\sup _{n \leq N} \mid \sum_{k=0}^{n-1}\left\langle X_{k \alpha}^{\varepsilon}-\bar{X}_{k \alpha}^{\varepsilon}, \int_{k \alpha}^{(k+1) \alpha}\left(\sigma\left(X_{s}^{\varepsilon}\right)-\sigma\left(\bar{X}_{k \alpha}^{\varepsilon}\right)\right) d B_{s}\right\rangle\right)^{\frac{p}{2}}\right] .
\end{aligned}
$$

We firstly estimate the term $\mathbb{E}\left[\sup _{k \alpha \leq t \leq(k+1) \alpha}\left|X_{t}^{\varepsilon}-X_{k \alpha}^{\varepsilon}\right|^{p}\right]$. Using the definition of $X^{\varepsilon}$, the Lipschitz property of $b, \beta_{\varepsilon}, \sigma$ and the proposition 3.1, we have

$$
\mathbb{E}\left[\sup _{k \alpha \leq t \leq(k+1) \alpha}\left|X_{t}^{\varepsilon}-X_{k \alpha}^{\varepsilon}\right|^{p}\right]+\mathbb{E}\left[\sup _{k \alpha \leq s \leq(k+1) \alpha}\left|X_{s}^{\varepsilon}-X_{(k+1) \alpha}^{\varepsilon}\right|^{p}\right] \leq \frac{c \alpha^{p}}{\varepsilon^{p}}+c \alpha^{\frac{p}{2}}
$$

Using this estimation, the Burkholder-Davis-Gundy inequality and the Lipschitz property of $\sigma$, we have the following estimate for the second term on the right hand side of (35):

$$
\begin{aligned}
& c N^{\frac{p}{2}-1} \sum_{k=0}^{N-1} \mathbb{E}\left[\left|\int_{k \alpha}^{(k+1) \alpha}\left(\sigma\left(X_{s}^{\varepsilon}\right)-\sigma\left(\bar{X}_{k \alpha}^{\varepsilon}\right)\right) d B_{s}\right|^{p}\right] \\
& \leq c\left(\frac{T}{\alpha}\right)^{\frac{p}{2}-1} \sum_{k=0}^{N-1} \alpha^{\frac{p}{2}} \mathbb{E}\left[\left|X_{k \alpha}^{\varepsilon}-\bar{X}_{k \alpha}^{\varepsilon}\right|^{p}+\sup _{k \alpha \leq s \leq(k+1) \alpha}\left|X_{s}^{\varepsilon}-X_{k \alpha}^{\varepsilon}\right|^{p}\right] \\
& \leq c \sum_{k=0}^{N-1} \alpha \mathbb{E}\left[\sup _{i \leq k}\left|X_{i \alpha}^{\varepsilon}-\bar{X}_{i \alpha}^{\varepsilon}\right|^{p}\right]+\left(\frac{c \alpha^{p}}{\varepsilon^{p}}+c \alpha^{\frac{p}{2}}\right) .
\end{aligned}
$$


We now consider the last term on the right hand side of (35). By the Burkholder-Davis-Gundy and Cauchy inequalities, we obtain

$$
\begin{aligned}
& c \mathbb{E}\left[\sup _{n \leq N}\left|\sum_{k=0}^{n-1} \int_{k \alpha}^{(k+1) \alpha}\left\langle X_{k \alpha}^{\varepsilon}-\bar{X}_{k \alpha}^{\varepsilon},\left(\sigma\left(X_{s}^{\varepsilon}\right)-\sigma\left(\bar{X}_{k \alpha}^{\varepsilon}\right)\right) d B_{s}\right\rangle\right|^{\frac{p}{2}}\right] \\
& \leq \frac{1}{2} \mathbb{E}\left[\sup _{n \leq N}\left|X_{n \alpha}^{\varepsilon}-\bar{X}_{n \alpha}^{\varepsilon}\right|^{p}\right]+2 c \mathbb{E}\left[\left(\sum_{k=0}^{N-1} \int_{k \alpha}^{(k+1) \alpha}\left|\sigma\left(X_{s}^{\varepsilon}\right)-\sigma\left(\bar{X}_{k \alpha}^{\varepsilon}\right)\right|^{2} d s\right)^{\frac{p}{2}}\right] .
\end{aligned}
$$

From the Lipschitz property of $\sigma,(31)$ and (36), we deduce that

$$
\begin{aligned}
& c \mathbb{E}\left[\left(\sum_{k=0}^{N-1} \int_{k \alpha}^{(k+1) \alpha}\left|\sigma\left(X_{s}^{\varepsilon}\right)-\sigma\left(\bar{X}_{k \alpha}^{\varepsilon}\right)\right|^{2} d s\right)^{\frac{p}{2}}\right] \\
& \leq \quad c N^{\frac{p}{2}-1} \alpha^{\frac{p}{2}} \sum_{k=0}^{N-1} \mathbb{E}\left[\left|X_{k \alpha}^{\varepsilon}-\bar{X}_{k \alpha}^{\varepsilon}\right|^{p}\right]+c N^{\frac{p}{2}-1} \alpha^{\frac{p}{2}} \sum_{k=0}^{N-1} \mathbb{E}\left[\sup _{k \alpha \leq s \leq(k+1) \alpha}\left|X_{s}^{\varepsilon}-X_{k \alpha}^{\varepsilon}\right|^{p}\right] \\
& \leq \quad c \sum_{k=0}^{N-1} \alpha \mathbb{E}\left[\sup _{i \leq k}\left|X_{i \alpha}^{\varepsilon}-\bar{X}_{i \alpha}^{\varepsilon}\right|^{p}\right]+\left(\frac{c \alpha^{p}}{\varepsilon^{p}}+c \alpha^{\frac{p}{2}}\right) .
\end{aligned}
$$

Hence

$$
\begin{aligned}
& c \mathbb{E}\left[\sup _{n \leq N}\left|\sum_{k=0}^{n-1} \int_{k \alpha}^{(k+1) \alpha}\left\langle X_{k \alpha}^{\varepsilon}-\bar{X}_{k \alpha}^{\varepsilon},\left(\sigma\left(X_{s}^{\varepsilon}\right)-\sigma\left(\bar{X}_{k \alpha}^{\varepsilon}\right)\right) d B_{s}\right\rangle\right|^{\frac{p}{2}}\right] \\
& \leq \frac{1}{2} \mathbb{E}\left[\sup _{n \leq N}\left|X_{n \alpha}^{\varepsilon}-\bar{X}_{n \alpha}^{\varepsilon}\right|^{p}\right]+c \sum_{k=0}^{N-1} \alpha \mathbb{E}\left[\sup _{i \leq k}\left|X_{i \alpha}^{\varepsilon}-\bar{X}_{i \alpha}^{\varepsilon}\right|^{p}\right]+\left(\frac{c \alpha^{p}}{\varepsilon^{p}}+c \alpha^{\frac{p}{2}}\right) .
\end{aligned}
$$

Using(36)-(38), inequality (35) becomes

$$
\mathbb{E}\left[\sup _{n \leq N}\left|X_{n \alpha}^{\varepsilon}-\bar{X}_{n \alpha}^{\varepsilon}\right|^{p}\right] \leq c \sum_{k=0}^{N-1} \alpha \mathbb{E}\left[\sup _{i \leq k}\left|X_{i \alpha}^{\varepsilon}-\bar{X}_{i \alpha}^{\varepsilon}\right|^{p}\right]+c \frac{\alpha^{\frac{p}{2}}}{\varepsilon^{p}},
$$

We use the Gronwall inequality to complete the proof.

\section{Remark:}

i) With the main result of the previous section, we finally deduce that

$$
\mathbb{E}\left[\sup _{n \leq N}\left|X_{n \alpha}-\bar{X}_{n \alpha}^{\varepsilon}\right|^{p}\right] \leq c \varepsilon^{\frac{p}{8}}+c \frac{\alpha^{\frac{p}{2}}}{\varepsilon^{p}} .
$$

ii) We may also obtain the almost sure uniform convergence of $\bar{X}_{n \alpha}^{\varepsilon}$ to $X_{t}^{\varepsilon}$, and of $\bar{X}_{t}^{\varepsilon}$ to $X_{t}$. 


\section{Applications}

\subsection{Some models}

Let $d \in \mathbb{N}^{*}, 0<M \leq \infty$ and $I=(0, M)$. We define a convex function $\phi: \mathbb{R} \rightarrow(-\infty,+\infty]$ of class $C^{1}(I)$ satisfying $\phi(x)=+\infty \quad \forall x \in I^{c} \cup\{0+\}$ and $\phi(M-)=+\infty$ if $M<$ $+\infty$. We put

$$
D=\left\{x=\left(x^{1}, x^{2}, \ldots, x^{d}\right): x^{1}<x^{2}<\ldots<x^{d}<x^{1}+M\right\}
$$

and define the function $\varphi: \mathbb{R}^{d} \rightarrow(\infty,+\infty]$ by

$$
\varphi(x)= \begin{cases}\sum_{\substack{1 \leq i<j \leq d \\+\infty}} \phi\left(x^{j}-x^{i}\right) & \text { on } D \\ & \text { on } D^{c} .\end{cases}
$$

It is easy to see that $\varphi$ is convex, 1.s.c., proper with $\operatorname{dom}(\varphi)=D$. E. Cépa and D. Lépingle [11] have shown that in this case the multivalued stochastic differential equation (9) turns into the stochastic differential equation (12).

We are interested in the following cases. Let $\gamma$ be a strictly positive real number and $B$ be a $d$-dimensional Brownian motion. With the initial condition $X_{0} \in \bar{D}$ a.s.:

for $\phi(x)=-\gamma \log (x)$ on $I=(0, \infty)$, let

$$
d X_{t}^{i}=d B_{t}^{i}+\gamma \sum_{1 \leq i \neq j \leq d} \frac{d t}{X_{t}^{i}-X_{t}^{j}} \quad X_{t}^{1} \leq X_{t}^{2} \leq \ldots \leq X_{t}^{d} ;
$$

$$
\begin{aligned}
& \text { for } \phi(x)=-\gamma \log \left(\sin \left(\frac{x}{2}\right)\right) \text { on } I=(0,2 \pi) \text {, let } \\
& d X_{t}^{i}=d B_{t}^{i}+\frac{\gamma}{2} \sum_{1 \leq i \neq j \leq d} \cot \left(\frac{X_{t}^{i}-X_{t}^{j}}{2}\right) d t \quad X_{t}^{1} \leq \ldots \leq X_{t}^{d} \leq X_{t}^{1}+2 \pi ;
\end{aligned}
$$

for $\phi(x)=-\gamma \log (\sinh (x))$ on $I=(0, \infty)$, let

$$
d X_{t}^{i}=d B_{t}^{i}+\gamma \sum_{1 \leq i \neq j \leq d} \operatorname{coth}\left(X_{t}^{i}-X_{t}^{j}\right) d t \quad X_{t}^{1} \leq X_{t}^{2} \leq \ldots \leq X_{t}^{d}
$$

Each system has a unique strong solution. We may find detailed studies of the system (40) in [5] and [10], of the systems (41) and (42) in [11].

The discretization error is $\frac{\sqrt{\alpha}}{\varepsilon}$ where $\varepsilon$ is the Yosida approximation parameter of $\varphi, \alpha=\frac{T}{N}$ is the step size of the Euler semi-implicit scheme; we must add the error term due the penalization approximation (see (39)) and the error term from the numerical computation. 


\subsection{Law support of the process on $[0, T]$}

Before proposing the simulations of process trajectories, it is interesting obtaining a support result for this trajectories. Recall firstly the formulation of the Skorohod multivalued problem given in [9]. Let $A$ be a multivalued maximal monotone operator and $w \in C\left([0, T] ; \mathbb{R}^{d}\right)$ verifying $w(0) \in \overline{D(A)}$. There exists an unique pair $(x, k)$ such that:

i) $x=\{x(t) ; 0 \leq t \leq T\}$ is continuous and taking values in $\overline{D(A)}$;

ii) $k=\{k(t) ; 0 \leq t \leq T\}$ is continuous with variation bounded, taking values in $\mathbb{R}^{d}$ and null at 0 ;

iii) for every pair of continuous functions $(\alpha, \beta)$ on $[0, T]$ and taking values in $\mathbb{R}^{d}$ such that $(\alpha(u), \beta(u)) \in G r(A) \forall u \in[0, T]$, the measure $\langle x(u)-\alpha(u), d k(u)-\beta(u) d u\rangle$ is positive. In the same paper, it is proved that for every $x_{0} \in \overline{D(A)}$, the application

$$
\Gamma: C_{x_{0}}\left([0, T] ; \mathbb{R}^{d}\right) \longrightarrow C_{x_{0}}([0, T] ; \overline{D(A)})
$$

is continuous, where $C_{x_{0}}\left([0, T] ; \mathbb{R}^{d}\right)$ is the set of continuous functions with initial value $x_{0}$.

If we now assume there exists a convex function $\varphi$ of the class $C^{1} \operatorname{Int}(\operatorname{dom}(\partial \varphi))$ such that $A=\partial \varphi$, then from the proof of Lemma 3.4 in [10] there exists a continuous function with finite variation $l$ verifying $l(0)=0$ and

$$
\begin{aligned}
& x(t)=w(t)-\int_{0}^{t} \nabla \varphi(x(s)) \mathbb{I}_{\{x(s) \in \operatorname{Int}(\operatorname{dom}(\partial \varphi))\}} d s+l_{t} \\
& \mathbb{I}_{\{x(t) \in \operatorname{Int}(\operatorname{dom}(\partial \varphi))\}} d l_{t}=0 .
\end{aligned}
$$

Proposition 5.1 With the previous conditions on $\varphi$, if $x_{0} \in \operatorname{Int}(\operatorname{dom}(\partial \varphi))$ and the space $C_{x_{0}}\left([0, T] ; \mathbb{R}^{d}\right)$ is equipped with the Wiener measure $\mathbb{P}_{x_{0}}$ starting from $x_{0}$, then the law support of $\Gamma$ is exactly the space $C_{x_{0}}([0, T] ; \overline{\operatorname{dom}(\partial \varphi)})$.

Proof: It is evident that the law support of $\Gamma$ is a subset of $C_{x_{0}}([0, T] ; \overline{\operatorname{dom}(\partial \varphi)})$.

Conversely, let $x \in C_{x_{0}}([0, T] ; \overline{\operatorname{dom}(\partial \varphi)})$ and $a \in \operatorname{Int}(\operatorname{dom}(\partial \varphi))$. For any $\varepsilon>0$, let $c>0$ with $c T \leq 1$ such that

$$
x^{\prime}(t)=a+(1-c t)(x(t)-a) \quad 0 \leq t \leq T
$$

belongs to $C_{x_{0}}([0, T] ; \operatorname{Int}(\operatorname{dom}(\partial \varphi))) \cap B(x, \varepsilon)$.

Put

$$
w^{\prime}(t)=x^{\prime}(t)+\int_{0}^{t} \nabla \varphi\left(x^{\prime}(s)\right) d s \quad 0 \leq t \leq T .
$$

Then $w^{\prime} \in C_{x_{0}}\left([0, T] ; \mathbb{R}^{d}\right)$ and it is clear by uniqueness of the solution of the Skorohod problem, that $x^{\prime}=\Gamma\left(w^{\prime}\right)$. By continuity of $\Gamma, \Gamma(B(x, \varepsilon))$ contains a ball $B\left(w^{\prime}, \eta\right)$ with $\eta>0$ and the properties of the Wiener measure entail

$$
\mathbb{P}_{x_{0}}\left(\Gamma(B(x, \varepsilon)) \geq \mathbb{P}_{x_{0}}\left(\Gamma\left(B\left(w^{\prime}, \eta\right)\right)>0\right.\right.
$$

which completes the proof.

It has been proved in [9] that the probability measure $\Gamma\left(\mathbb{P}_{x_{0}}\right)$ is exactly the law of the process $X$ in (9) with $b=0, \sigma=I$ and $X_{0}=x_{0}$. 


\subsection{Linearization method}

We firstly consider the process $\mathbb{R}_{+}$, called the Bessel process with dimension $2 \gamma+1$ that is the solution to the one-dimensional equation

$$
d X_{t}=d B_{t}+\gamma \frac{d t}{X_{t}}
$$

We propose a linearization method where the Brownian motion is approximated by a piecewise linear path $w$ defined for every $t \in[n \alpha,(n+1) \alpha), 0 \leq n \leq N-1$, by

$$
B_{t} \simeq w(t)=\frac{B_{(n+1) \alpha}-B_{n \alpha}}{\alpha} t+\left((n+1) B_{n \alpha}-n B_{(n+1) \alpha}\right) .
$$

The equation (43) becomes, for every $t \in[n \alpha,(n+1) \alpha), 0 \leq n \leq N-1$,

$$
d \bar{X}_{t}=\left(\frac{B_{(n+1) \alpha}-B_{n \alpha}}{\alpha}+\frac{\gamma}{\bar{X}_{t}}\right) d t .
$$

Since the Brownian motion has independent increments, the approximate value $\bar{X}_{n+1}$ (at time $(n+1) \alpha)$ satisfies

$$
\left(\bar{X}_{n+1}-\bar{X}_{n}\right) Y_{n+1}-\gamma \ln \left(\frac{\bar{X}_{n+1} Y_{n+1}+\gamma}{\bar{X}_{n} Y_{n+1}+\gamma}\right)=\alpha Y_{n+1}^{2}
$$

where $\left(Y_{n}\right)_{1 \leq n<N-1}$ are i.i.d. $\mathcal{N}(0, \sqrt{\alpha})$ random variables. This equation has one or two positive roots $\bar{X}_{n+1}$. We choose the largest one. The error order of this method is smaller than $\frac{1}{2}$. Indeed, by Theorem 7.1 in [9] we have

$$
\sup _{t \leq T}\left|X_{t}-\bar{X}_{t}\right| \leq 2 \sup _{t \leq T}\left|B_{t}-w(t)\right| \quad \text { a.s. }
$$

The error order is estimated thanks to the following proposition ( we may find a proof of in [18]):

Proposition 5.2 There exists a positive $c$ such that, for every $\alpha \in(0,1)$,

$$
\mathbb{E}\left[\sup _{n \leq N} \sup _{n \alpha \leq t \leq(n+1) \alpha}\left|B_{t}-B_{n \alpha}\right|^{2}\right] \leq c \alpha \log \left(\frac{1}{\alpha}\right)
$$

\subsection{Penalization method}

1. For the one-dimensional equation (43), we have the explicit formula of the Yosida approximation of $\partial \varphi$

$$
\beta_{\varepsilon}(x)=\frac{x-\sqrt{x^{2}+4 \varepsilon \gamma}}{2 \varepsilon} .
$$

Using the semi-implicit scheme (26) we get the explicit expression (from now on we write $\bar{X}_{n}^{\varepsilon}$ in place of $\bar{X}_{n \alpha}^{\varepsilon}$ )

$$
\bar{X}_{n+1}^{\varepsilon}=\frac{(2 \varepsilon+\alpha)\left(\bar{X}_{n}^{\varepsilon}+Y_{n+1}\right)+\alpha \sqrt{\left(\bar{X}_{n}^{\varepsilon}+Y_{n+1}\right)^{2}+4 \gamma(\varepsilon+\alpha)}}{2(\varepsilon+\alpha)},
$$


where $\left(Y_{n}\right)_{1 \leq n \leq N}$ are i.i.d. $\mathcal{N}(0, \sqrt{\alpha})$ variables.

2. In the multidimensional case or in the one-dimendional case with cot or coth, we do not have any explicit formula for the Yosida approximation of $\partial \varphi$ but we can program a numerical computation of $\beta_{\varepsilon}$ (see the Annex). After obtaining $\beta_{\varepsilon}$, at the $(n+1)$-th step, we solve the equation

$$
\bar{X}_{n+1}^{\varepsilon}=\bar{X}_{n}^{\varepsilon}-\alpha \beta_{\varepsilon}\left(\bar{X}_{n+1}^{\varepsilon}\right)+Y_{n+1}
$$

where $\left(Y_{n}\right)_{1 \leq n \leq N}=\left(Y_{n}^{1}, \ldots, Y_{n}^{d}\right)_{1 \leq n \leq N}$ are independent r.v. with i.i.d. with $\mathcal{N}(0, \sqrt{\alpha})$ components .

Since $\beta_{\varepsilon}$ is Lipschitz with constant $\frac{1}{\varepsilon}$, if $\alpha$ is much smaller than $\varepsilon$, the function $-\alpha \beta_{\varepsilon}(x)+$ $Y_{n+1}+\bar{X}_{n}^{\varepsilon}$ is Lipschitz with constant $\frac{\alpha}{\varepsilon}<1$. By the fixed-point theorem the following algorithm:

$$
\begin{aligned}
& \text { first step: } \bar{X}_{n+1,1}^{\varepsilon}=-\alpha \beta_{\varepsilon}\left(\bar{X}_{n}^{\varepsilon}\right)+Y_{n+1}+\bar{X}_{n}^{\varepsilon} \\
&(k+1) \text { - th step: } \quad \bar{X}_{n+1, k+1}^{\varepsilon}=-\alpha \beta_{\varepsilon}\left(\bar{X}_{n+1, k}^{\varepsilon}\right)+Y_{n+1}+\bar{X}_{n}^{\varepsilon}
\end{aligned}
$$

converges quickly to the solution $\bar{X}_{n+1}^{\varepsilon}$ of (45). In each loop, we use the Newton method with constraints (see the annex) to calculate $\beta_{\varepsilon}\left(\bar{X}_{n+1, k}^{\varepsilon}\right)$.

In [10], the authors have proved existence of simple collisions ( two particles at the same place) if and only if $\gamma<\frac{1}{2}$ in the case (40) and (41), and existence of simple collisions with positive probability if and only if $\gamma<\frac{1}{2}$ in the case (42). Fig 1 displayss the simulated trajectories of five particles $(d=5)$ following equation (40) with $\gamma=0.25$ and Fig 2 displays the same system with $\gamma=1$.

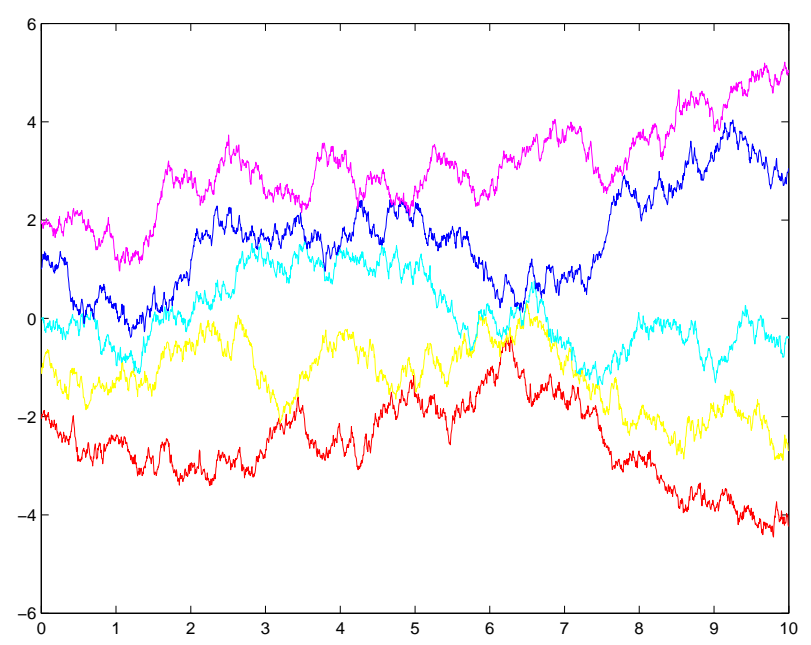

FIG 1. - Paths of five particles from the system (40) with collisions. 


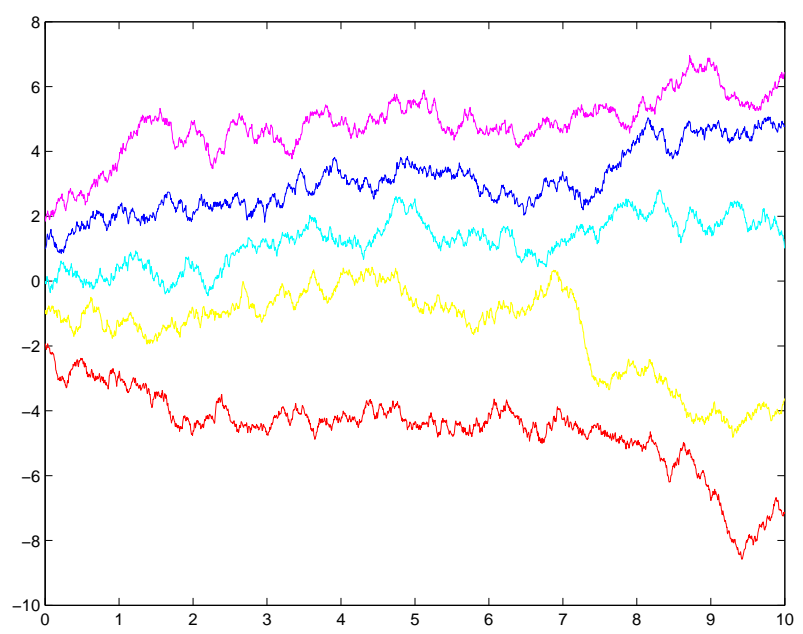

FIG 2. - Paths of five particles from the system (40) without collisions.

\subsection{Some properties of particles}

1. In the case (40), we define the function $f: \mathbb{R}^{d} \rightarrow \mathbb{R}$ by

$$
f(x)=\frac{1}{2 d} \sum_{i, j=1}^{d}\left(x^{i}-x^{j}\right)^{2} .
$$

Applying the Ito formula and after some computation as in [12], p.251, we get

Proposition $5.3\left(f\left(X_{t}\right)\right)_{t \geq 0}$ is a Bessel square with dimension $(d-1)(d \gamma+1)$.

Remark: Other functions have an analogous property, for example

$$
f(x)=\sum_{i=1}^{d}\left(x^{i}\right)^{2} .
$$

Thanks to the previous proposition, we can compute exactly the expectation of $f\left(X_{t}\right)$. On the other hand, we calculate the expectation of $f\left(\bar{X}_{t}^{\varepsilon}\right)$ by the Monte-Carlo method. The a.s. uniform convergence of $\bar{X}_{t}^{\varepsilon}$ to $X_{t}$ allows us to test the proposed method. For $d=3$ and $\gamma=1$, Fig 3 compares a Monte Carlo simulation of $f\left(\bar{X}_{t}^{\varepsilon}\right)$ with the theoritical value $(d-1)(d \gamma+1) t$. 


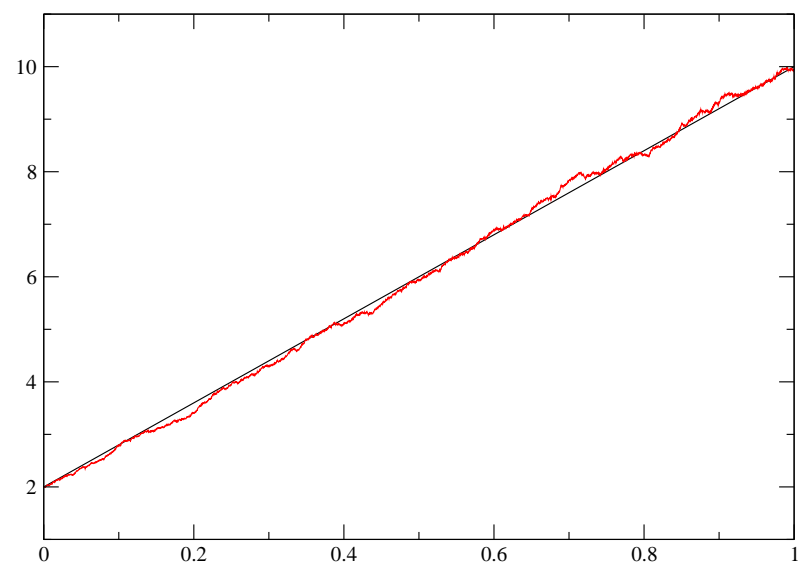

FIG 3. - Exact expectation and Monte-Carlo approximation.

2. We can find the proof of the following theorem in [17] which presents the asymptotic behavior of particles with hyperbolic interaction.

Proposition 5.4 Let $\gamma \geq \frac{1}{2}$. For the particles of system (42) we have

$$
\lim _{t \rightarrow \infty} \frac{X_{t}^{i}}{t}=2 \gamma i-\gamma(d+1) \quad \text { a.s. } \quad \forall 1 \leq i \leq d .
$$

Fig 4 displays a simulation with $d=3$ and $\gamma=1$.

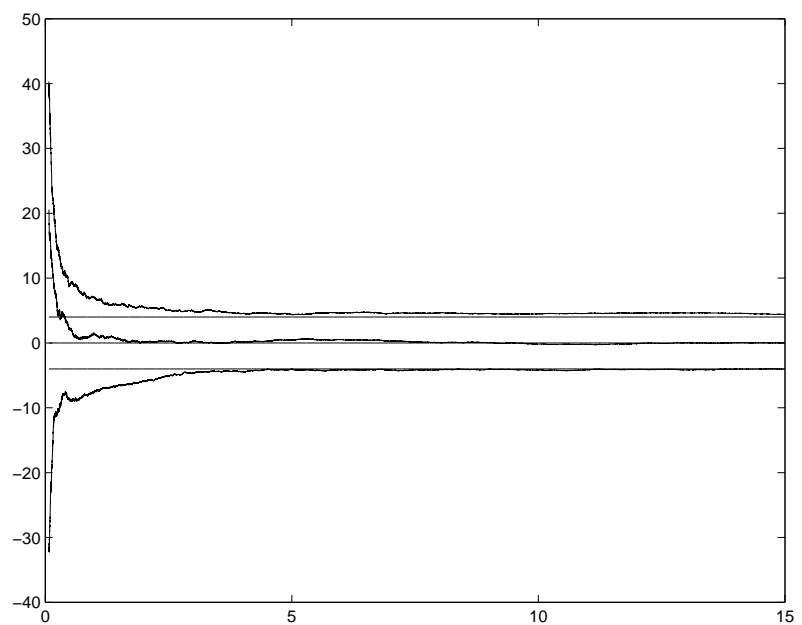

FIG 4. - Asymptotic behavior of the system(42).

\section{ANNEX}

To obtain the approximate value $\bar{X}^{\varepsilon}$ at each time step, we need to solve the equation

$$
x=-\alpha \beta_{\varepsilon}(x)+c,
$$


where $c$ is the sum of the previous approximate value and a r.v. $Y=\left(Y^{1}, \ldots, Y^{d}\right) \in \mathbb{R}^{d}$ (the choice of $Y$ on each step is independent) whose components are Gaussian centered with variance $\alpha$ in $\mathbb{R}$ and $\beta_{\varepsilon}$ is the Yosida approximation of subdifferential of $\varphi$.

Since $\beta_{\varepsilon}$ is Lipschitz with constant $\frac{1}{\varepsilon}$, if we choose $\alpha<\varepsilon$, then the function $-\alpha \beta_{\varepsilon}$ is Lipschitz continuous with constant smaller than 1 . The algorithm

$$
\begin{aligned}
& x^{(0)} \\
& x^{(k+1)}=-\alpha \beta_{\varepsilon}\left(x^{(k)}\right)+c
\end{aligned}
$$

converges quickly to the solution of equation (47).

We calculate $\beta_{\varepsilon}$ by the formula

$$
\beta_{\varepsilon}(x)=\frac{x-\pi_{\varepsilon} x}{\varepsilon} \quad, \quad x \in \mathbb{R}^{d}
$$

where $\pi_{\varepsilon} x \in \operatorname{Int}(\operatorname{dom}(\varphi))$ and minimizes the function

$$
f(y)=\frac{1}{2 \varepsilon}|x-y|^{2}+\varphi(y) \quad, \quad y \in \mathbb{R}^{d} .
$$

Since $\varphi$ is proper l.s.c. convex, we use the Newton method with convex constraints (see [7]). Consider for example the system (40). We must solve the problem $(P 1)$ :

$$
\begin{aligned}
& \min _{y \in D} f(y)=\frac{1}{2 \varepsilon}|x-y|^{2}-\gamma \sum_{i=1}^{d-1} \sum_{j=i+1}^{d} \ln \left(y_{j}-y_{i}\right) \\
& x \in \mathbb{R}^{d}, \varepsilon \in \mathbb{R}_{+}^{*}, \gamma \in \mathbb{R}_{+}^{*} .
\end{aligned}
$$

The function $f$ is strictly convex, twice differentiable and tends to infinity on the boundary of the domain, the problem $(P 1)$ has a unique solution $\bar{x} \in D$ satisfying

$$
\left\{\begin{array}{l}
\bar{x}_{i}-x_{i}-\varepsilon \gamma \sum_{j \neq i} \frac{1}{\bar{x}_{j}-\bar{x}_{i}}=0 \quad \forall i=1, \ldots, d \\
\bar{x}=\left(\bar{x}_{1}, \ldots, \bar{x}_{d}\right) \in D .
\end{array}\right.
$$

Putting

$$
\begin{aligned}
\bar{y}_{(i-1) d-\frac{i(i-1)}{2}+j-i} & =\bar{x}_{j}-\bar{x}_{i} & & \forall 1 \leq i<j \leq d ; \\
\bar{s}_{k} & =\frac{\varepsilon \gamma}{\bar{y}_{k}} & & \forall 1 \leq k \leq \widetilde{d}, \quad \tilde{d}=\frac{d(d-1)}{2},
\end{aligned}
$$

the problem $(P 1)$ becomes

$$
\left\{\begin{aligned}
\bar{x}_{i}-x_{i}-\sum_{j<i} \bar{s}_{(j-1) d-\frac{j(j-1)}{2}+i-j}+\sum_{j>i} \bar{s}_{(i-1) d-\frac{i(i-1)}{2}+j-i}=0 & \forall i=1, \ldots, d \\
\bar{x}_{i}-\bar{x}_{j}+\bar{y}_{(i-1) d-\frac{i(i-1)}{2}+j-i}=0 & \forall 1 \leq i<j \leq d \\
\bar{y}_{i} \bar{s}_{i}-\varepsilon \gamma=0 & \forall 1 \leq i \leq \widetilde{d} \\
\bar{y}_{i}>0 & \forall 1 \leq i \leq \tilde{d} \\
\bar{s}_{i}>0 & \forall 1 \leq i \leq \tilde{d} .
\end{aligned}\right.
$$




\section{References}

[1] I. Asiminonei-A. Rascanu. Approximation and Simulation of Stochastic Variational Inequalities - Splitting up Method. Numer. Funct. Anal. and Optimiz. 18, 231-282 (1997).

[2] V. Barbu et Th. Precupanu. Convexity and Optimization in Banach Spaces. Second edition, Editura Academiei (1986).

[3] A. Bensoussan-A. Rascanu. Stochastic Variational Inequalities in Infinite Dimensional Space. Numer. Funct. Anal. and Optimiz. 18, 19-54 (1997).

[4] F. Bernardin. Multivalued stochastic differential equations: convergence of a numerical scheme. Set-Valued Anal 11, 393-415 (2003).

[5] A. Bonami, F.Bouchut, E.CÉPa, D. Lépingle. A nonlinear SDE involving Hilbert transform. Journal of Function Analysis 2, 390-406 (1999).

[6] H. BRÉZis. Opérateurs monotones et semi-groupes de contractions dans les espaces de Hilbert. North Holland (1973).

[7] J. CÉA. Optimisation Théorie et Algorithmes. Dunod (1971).

[8] E. CÉPA. Equations différentielles stochastiques multivoques. Séminaire Probabilités XXIX, 86-107 (1995).

[9] E. CÉPA. Problème de Skorohod multivoque. The Annals of Probability 26, 500-532 (1998).

[10] E. CÉPA, D.LÉPingle. Diffusing particles with electrostatic repulsion. Probab. Theory Relat. Fields 107, 429-449 (1997).

[11] E. CÉPA, D.LÉPingle. Brownian particles with electrostatic repulsion on the circle: Dyson's model for unitary random matrices revisited. ESAIM Proba. Statist. 5, 203-224 (2001).

[12] T. Chan. The Wigner semi-circle law and eigenvalues of matrix-valued diffusions. Theory Rel. Fields 93, 249-272 (1992).

[13] A. Diop. Sur la discrétisation et le comportement à petit bruit d'EDS unidimensionnelles dont les coefficients sont à dérivées singulières. Ph. D. (2003). 
[14] Y. Z. Hu. Semi-implicit Euler-Maruyama scheme for stiff stochastic equations. Progr. Proba. 38, 183-202 (1996).

[15] P. E. Kloeden, E. Platen. Numerical Solution of Stochastic Differential Equations. Springer-Verlag (1992).

[16] P. KRÉE. Diffusion Equation for Multivalued Stochastic Differential Equations. J. Funct. Anal. 49, 73-90 (1982).

[17] J. R. Norris,L. C. G. Rogers, D. Williams. Brownian motions of ellipsoids. Prob. Th. Rel. Fields, 95 (1986).

[18] R. Pettersson. Approximations for stochastic differential equations with reflecting convex boundaries. Stochastic Process. Appl. 59, 757-765 (1995).

[19] R. Pettersson. Projection scheme for stochastic differential equations with convex constraints. Stochastic Process. Appl. 88, 125-134 (2000).

[20] L. Slominski. On approximations of solutions of multidimentional SDE's with reflecting boundary conditions. Stochastic Process. Appl. 50, 179-219 (1994).

[21] A. Storm. Stochastic differential equations with convex constraint. Stochastics and Stochastics Reports 53, 241-274 (1995).

[22] H. TANAKA. Stochastic differential equations with reflecting boundary conditions in convex regions. Hiroshima Math. J. 9, 163-177 (1979). 